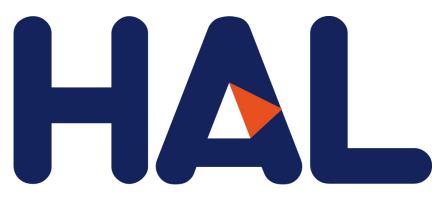

archives-ouvertes

\title{
What Thermal Analysis Can Tell Us About Melting of Semicrystalline Polymers: Exploring the General Validity of the Technique
}

\author{
Alexey Melnikov, Martin Rosenthal, Dimitri Ivanov
}

\section{To cite this version:}

Alexey Melnikov, Martin Rosenthal, Dimitri Ivanov. What Thermal Analysis Can Tell Us About Melting of Semicrystalline Polymers: Exploring the General Validity of the Technique. ACS Macro Letters, Washington, D.C: American Chemical Society, 2018, 7 (12), pp.1426-1431. 10.1021/acsmacrolett.8b00754 . hal-02415172

\section{HAL Id: hal-02415172 \\ https://hal.archives-ouvertes.fr/hal-02415172}

Submitted on 7 Jan 2021

HAL is a multi-disciplinary open access archive for the deposit and dissemination of scientific research documents, whether they are published or not. The documents may come from teaching and research institutions in France or abroad, or from public or private research centers.
L'archive ouverte pluridisciplinaire HAL, est destinée au dépôt et à la diffusion de documents scientifiques de niveau recherche, publiés ou non, émanant des établissements d'enseignement et de recherche français ou étrangers, des laboratoires publics ou privés. 


\title{
What thermal analysis can tell us about melting of semicrystalline polymers: exploring the general validity of the technique
}

\author{
Alexey P. Melnikov, ${ }^{\dagger, a, b}$ Martin Rosenthal, $,^{\dagger},{ }^{*}, \mathrm{c}$ and Dimitri A. Ivanov ${ }^{*}, \mathrm{a}, \mathrm{b}, \mathrm{d}$ \\ ${ }^{a}$ Lomonosov Moscow State University, Faculty of Fundamental Physical and Chemical Engineering, GSP-1, 1-51 Leninskie \\ Gory, Moscow, Russian Federation; \\ ${ }^{\mathrm{b}}$ Moscow Institute of Physics and Technology (State University), Institutskiy per. 9, Dolgoprudny, Russian Federation; \\ ${ }^{\mathrm{c}}$ European Synchrotron Radiation Facility (ESRF), 6 rue Jules Horowitz, 38043 Grenoble, France \\ ${ }^{\mathrm{d}}$ Institut de Sciences de Matériaux de Mulhouse-IS2M, CNRS UMR 7361, Jean Starcky, 15, F-68057 Mulhouse, France.
}

KEYWORDS: semicrystalline morphology, ultrafast chip calorimetry, X-ray scattering, multiple melting, semirigid-chain polymers, poly(trimethylene terephthalate).

\begin{abstract}
Thermal characterization of semicrystalline polymers can constitute a difficult task due to the metastable nature of polymer crystals. It is well documented that polymer structure can reorganize during the thermoanalytical experiment. It has become also clear that thermal analysis alone cannot discriminate the reorganization processes from multiple melting events. Therefore, instead of studying the initial sample state the measurements may simply reflect the structural evolution uncontrollably occurring during the experiment. Here an original setup combining in-situ ultrafast chip calorimetry with millisecond time-resolved X-ray scattering is used to find the structural signature of the reorganization processes. The information is further used to construct the Heating-Rate versus Crystallization-Temperature Reorganization (HR-CT-R) diagram. The diagram allows designing experiments in which one can completely exclude uncontrolled evolution of the semicrystalline structure. For a typical aromatic polyester, poly(trimethylene terephthalate), the critical heating rate above which all reorganization processes cease to exist can reach $1,000 \mathrm{~K} / \mathrm{s}$ and more.
\end{abstract}

The thermal characterization of semicrystalline polymer materials can constitute a difficult experimental task due to the metastable nature of the semicrystalline polymer structure.[1,2] For the classical lamellar systems, polymer structure is composed of several-nanometer-thick crystalline lamellae interwoven with amorphous regions of a comparable thickness. One of the main problems in performing thermal studies on such systems is due to the fact that the semicrystalline structure can evolve in the course of the measurement. Therefore, instead of studying the initial sample state the experimental results may simply reflect the structural evolution uncontrollably occurring during the experiment. The problem has been recognized early on in studies of semirigid-chain semi-crystalline polymers such as poly(ethylene terephthalate), PET. The DSC heating traces of PET showed two and more melting peaks [3] that were interpreted as a manifestation of a melting-reorganization process operating during the experiment. Accordingly, the authors of work [3] pointed out that "the high temperature peak is due to a recrystallization and melting process taking place while the material is being scanned. Hence the DSC peaks are not a direct reflection of the state of the material at room temperature prior to the scan." In the following years, numerous studies of the thermal behavior of semirigid-chain polymers confirmed the general character of the double- or even multiple-melting behavior. Moreover, it was realized that "recrystallization is observed over the whole interval between $\mathrm{T}_{\mathrm{c}}$ and $\mathrm{T}_{\mathrm{m}}$ ".[2] Apart from the works on PET [4-5], the complex DSC traces with multiple exothermic and endothermic peaks have been recorded on many other polymers such as poly(ether ether ketone), PEEK [6-9], poly(trimethylene terephthalate), PTT [10-11], etc. Since it was not always known if the structural reorganization takes place during the reported experiments, the results of these studies remain ambiguous. It is therefore compulsory to rank the experimental conditions for example on a basis of a state diagram to appreciate if any structural reorganization operates during heating. Clearly, only experiments completely excluding such reorganization can provide information relevant to the initial sample state. Such diagram can then serve as a practical guide for materials scientists to define the conditions of meaningful thermal measurements.

In the past, several diagrams have been proposed to reflect the changes of material's state or structure in the course of isothermal annealings, as well as constant-rate heatings and coolings. They include Time-Temperature-Transformation (TTT)[12], Continuous Heating Transfor-mation (CHT), and Continuous Cooling Transformation (CCT) [13-14] diagrams 
and Temperature vs. heating rate transformation diagram [15], which were put forward to analyze the thermosetting resins hardening, solidification of metals and alloys, etc. In the case of semicrystalline polymers the critical experimental parameter is the heating rate. On the other hand, the pace at which the structural reorganization occurs for a given polymer at a given temperature is mainly dependent on the crystallization temperature, $\mathrm{T}_{\mathrm{c}}$. Therefore, the diagram in question should allow evaluating the critical heating rate for a chosen $T_{c}$. In the following, the critical heating rate stands for the smallest heating rate at which the reorganization ceases to operate during heating up to the final melting region. A meaningful thermoanalytical experiment has to be conducted at heating rates equal or higher than the critical heating rate corresponding to particular processing conditions. Hence, the discussed diagram should display the critical rate in the heating-rate versus crystallization-temperature coordinates, which makes it logical to be termed as Heating-Rate versus Crystallization-Temperature Reorganization Diagram (HR-CT-R).

The main problem in establishing such a diagram consists in the fact that the double- or multiple-melting patterns observed in the DSC traces of semicrystalline polymers require additional information for their interpretation. In this case, the calorimetry data alone is largely insufficient for such analysis. Moreover, in most instances the conventional calorimetry cannot provide the heating rates high enough to bypass the reorganization. This makes the use of the MEMS-based calorimetry, or nanocalorimetry [16], practically compulsory. Recently, we designed and tested an experimental setup combining in-situ ultrafast chip calorimetry and microfocus X-ray scattering.[17-20] Such real-time combination became possible thanks to the advent of a new-generation single-photon-counting fast X-ray detectors, which allowed to significantly accelerate this notoriously slow technique. We showed that the double-melting pattern per se does not yet mean that the reorganization occurs.[21] The dou- ble-melting behavior can be in many instances just a manifestation of the complex thermodynamics of polymer crystals that strongly interact with the neighboring amorphous regions.[2223 ] This can cause for example a significant depression of their melting transition temperatures due to the negative pressure effects.[24-25]

In the present work, the HR-CT-R diagram will be established for a typical semirigid-chain semicrystalline polymer, PTT, the complex supramolecular organization of which has been recently addressed.[26-28]. The scheme of the experimental setup combining ultrafast chip calorimetry and millisecond-time-resolved X-ray scattering is shown in Figure 1.

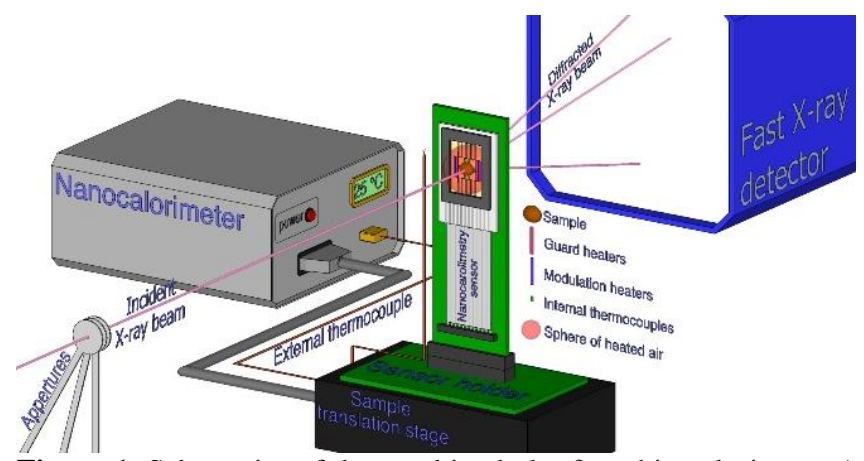

Figure 1. Schematics of the combined ultrafast chip calorimetry / micro-focus X-ray scattering setup installed at the ID13 microfocus beamline of ESRF. The incident X-ray beam is focused on the active region of the nanocalorimetric sensor down to the size of $14 \mathrm{x}$ $17 \mu \mathrm{m}^{2}$.

The setup was designed and installed at the micro-focus branch (EH3) of the ID13 beamline (ESRF, Grenoble). A custom-made modular nanocalorimeter sensor holder (cf. Figure 1) was positioned in the beam using sample translation stage allowing controlled and independent motion along $\mathrm{X}, \mathrm{Y}$ and $\mathrm{Z}$ axes with a step of less than $0.5 \mu \mathrm{m}$. Further experimental details can be found in our previous publications [17-20] and SI. 


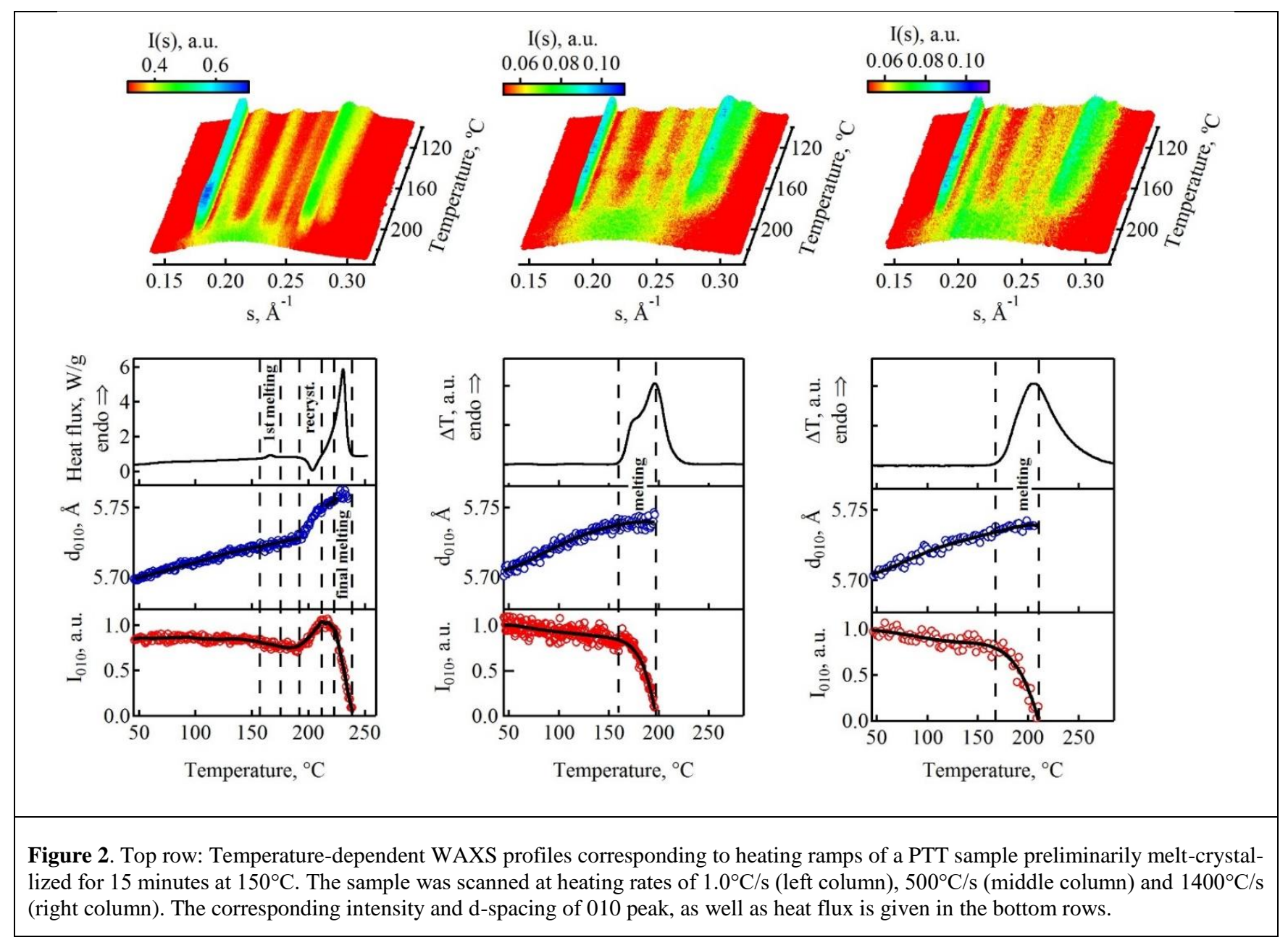

Figure 2 displays variable-temperature WAXS data together with the corresponding DSC and nanocalorimetric traces recorded during heating ramps at different heating rate. Importantly, all the experiments have been carried out on the samples isothermally melt-crystallized at $150^{\circ} \mathrm{C}$. The heating rates applied in the experiments increase from the left to right side of the figure. It can be seen that the recorded calorimetric curves crucially depend on the heating rate applied, which means that the experimentalist in this case is challenged to choose the conditions to acquire meaningful thermal data.

It is noteworthy that during the experiments with slow heating (i.e. at $1.0^{\circ} \mathrm{C} / \mathrm{s}$ ), the nanocalorimeter was operated in a temperature-modulation mode with a small temperature amplitude. For the sake of comparison, the graph displays an ex-situ measurement performed at the same rate on a power-compensated Perkin-Elmer DSC 8000 device. The DSC heating curve shown in the left column of the figure reveals low-temperature melting event followed by an exothermic recrystallization and final melting peak. The central and right columns contain in-situ nanocalorimetric data. The heating rates used in these columns are clearly beyond the reach of any classical DSC instrument and, therefore, required employment of a custom-built in-situ nanocalorimeter.[17]

The time- and temperature-resolved WAXS profiles shown in the top row of Figure 2 reveal a difference in the behavior of the strongest 010 peak of PTT located at $0.175 \AA^{-1}$. Thus, for the slowest heating ramp (cf. the top left panel of the figure) the intensity of the peak abruptly increases at ca. $200^{\circ} \mathrm{C}$, i.e. just before entering the final melting region. The temperature interval corresponding to this increase coincides with the recrystallization peak visible in the corresponding DSC trace. To better appreciate the behavior of the 010 peak, the temperature dependence of its intensity and $d$-spacing $\left(\mathrm{d}_{010}\right)$ is displayed in the two bottom rows of the figure. It can be seen that the reorganization event observable in the DSC trace coincides with the increase of the intensity and the corresponding $d$-spacing of the 010 peak. It is clear that these features reflect the formation of a new population of crystals stable at higher temperatures and each them can constitute the structural signature of a reorganization process.

The presence of reorganization during heating at $1.0^{\circ} \mathrm{C} / \mathrm{s}$ means that for $\mathrm{T}_{\mathrm{c}}$ of $150^{\circ} \mathrm{C}$ this heating rate is below the critical heating rate. In contrast, fast heating at $500^{\circ} \mathrm{C} / \mathrm{s}$ and $1400^{\circ} \mathrm{C} / \mathrm{s}$ do not exhibit signs of reorganization. For example, at these heating rates the temperature evolution of $\mathrm{d}_{010}$ follows an opposite trend, i.e. the $\mathrm{d}_{010}$ values display normal thermal expansion followed by leveling off in the melting region. Importantly, for a heating rate of $500^{\circ} \mathrm{C} / \mathrm{s}$ the nanocalorimetric trace reveals a complex shape typical of the classical double-melting behavior. The low-temperature melting peak is in this case visible as a shoulder of the main endotherm. Since there is no reorganization taking place at this heating rate, one can safely conclude 
that the complex shape of the melting peak such as the presence of two melting peaks does not necessarily imply structural reorganization. This confirms the general conclusions of our previous publications.[17,19,21] It is noteworthy that for the heating rate of $1400^{\circ} \mathrm{C} / \mathrm{s}$ the shape of the melting peak remains reminiscent of the double melting, i.e. asymmetric, but more smeared in comparison with the one at $500^{\circ} \mathrm{C} / \mathrm{s}$. This can be explained by convolution of the thermal signal with the timeresponse function of the nanocalorimetric sensor, which becomes more noticeable at higher heating rates.

Figure 3 displays the 1D SAXS curves (left column) measured during the in-situ experiments displayed in Figure 2. The corresponding 1D auto-correlation functions (CFs) are shown in the right column of the figure. The curves correspond to room temperature, onset of melting and a temperature near the maximum of the main melting peak. One can appreciate the shift of the main SAXS interference maximum to lower s-values with temperature, which reveals the changes occurring in the morphological parameters on heating.
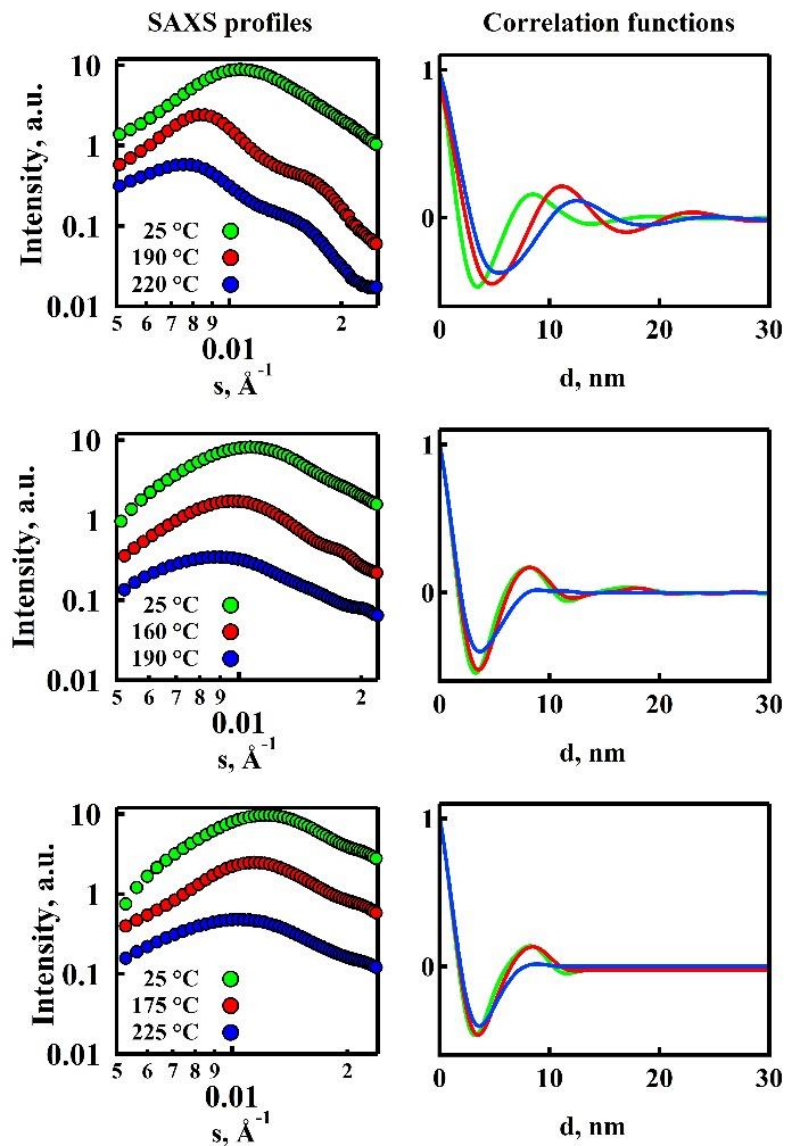

Figure 3. Selected SAXS curves (left) measured during the same experiments as in Figure 2. The applied heating rates are $1.0^{\circ} \mathrm{C} / \mathrm{s}$ (top), $500^{\circ} \mathrm{C} / \mathrm{s}$ (middle) and $1400^{\circ} \mathrm{C} / \mathrm{s}$ (bottom). The corresponding normalized 1D CFs are displayed in the right column of the figure.

The structural evolution can be further characterized with the help of the SAXS CFs. One can see that in the self-correlation triangle the CFs corresponding to the two fast heating rates behave similarly, whereas this is at variance for the slowest heating rate. The slopes of the CFs in the self-correlation triangle are in direct relationship to the smallest of the two SAXS distances, i.e. either crystal or amorphous layer thicknesses. Since PTT is a low-crystallinity polymer, the smallest SAXS distance is pertinent to crystal thickness. Therefore, the crystals remain invariable in thickness over the whole temperature range at heating rates of 500 and $1400^{\circ} \mathrm{C} / \mathrm{s}$. By contrast, crystal thickness increases with temperature for the heating rate of $1.0^{\circ} \mathrm{C} / \mathrm{s}$, which confirms that reorganization is indeed taking place in this case.

To find out the critical heating rates corresponding to other $\mathrm{T}_{\mathrm{c}}$ 's some additional experiments have been carried out. Figure 4 displays the in-situ nanocalorimetric curves measured for PTT melt-crystallized at 120,150 and $180^{\circ} \mathrm{C}$. In these experiments, heating rates of $1,30,60,125,500,1400$ and $3000^{\circ} \mathrm{C} / \mathrm{s}$ were applied. The bottom panels of the figure show the $\mathrm{d}_{010}$ values for each of the heating rates. For the sake of clarity, all nanocalorimetric curves and $d$-spacings are offset vertically with the respect to the curves measured at the lowest heating rate. To sort out the ramps for which the reorganization took place one can use the criterion of a rapid increase of $\mathrm{d}_{010}$. This feature is present in three slowest heating ramps for the left column and two slowest ramps of the middle column. Finally, only the slowest ramp displayed in the right column reveals the structural signature of reorganization. Interestingly, the cases where the reorganization did not happen correspond to the nanocalorimetric curves with just double melting. This holds for the curves for $\mathrm{T}_{\mathrm{c}}$ of $180^{\circ} \mathrm{C}$ in which the two melting peaks are located close to each other. In contrast, the cases with reorganization correspond to the nanocalorimetric curves revealing a pattern, which is more complex than just usual double melting. However, since none of the nanocalorimetric curves exhibits an exothermic event on heating, it would be anyhow problematic to identify the runs during which the reorganization did occur in the absence of the complementary in-situ X-ray data. 

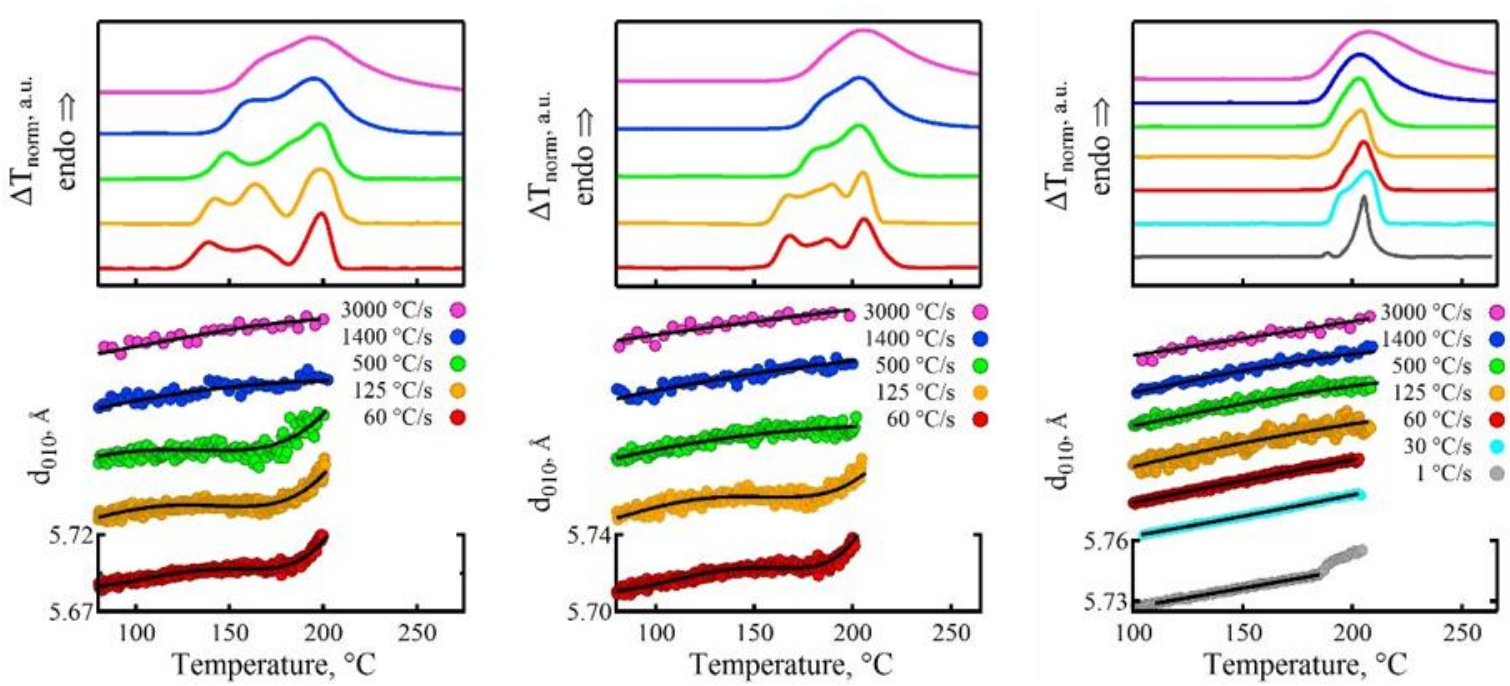

Figure 4. Nanocalorimetric curves (top panels) measured during heating of PTT samples isothermally melt-crystallized at 120 (left), 150 (middle) and $180^{\circ} \mathrm{C}($ right) at different heating rates. The corresponding temperature dependencies of do10 are displayed in the bottom panels. For the sake of clarity, the nanocalorimetric curves and $d$-spacing dependencies are offset vertically with the respect to the curves measured at the lowest heating rate. For the sake of comparison, the calorimetric curve at $1.0^{\circ} \mathrm{C} / \mathrm{s}$ was measured ex situ on a power-compensated Perkin-Elmer DSC 8000.

All the obtained data can be now merged on the HR-CT-R diagram shown in Figure 5. The filled and empty symbols mark the zones of the diagram where the reorganization is present and absent, respectively. The border between the zones is emphasized with a solid line, which constitutes the $\mathrm{T}_{\mathrm{c}}$-dependence of the critical heating rate. It can be seen that the latter significantly decreases with $T_{c}$, which can be explained by the fact that the reorganization rate significantly slows down when approaching the final melting. Eventually, at the highest $\mathrm{T}_{\mathrm{c}}$ 's the reorganization will not happen even at conventional-DSC heating rates. The proposed HR-CT-R diagram can be also established for any other semicrystalline polymer to be used as a guide in choosing proper conditions of thermal analysis experiments.

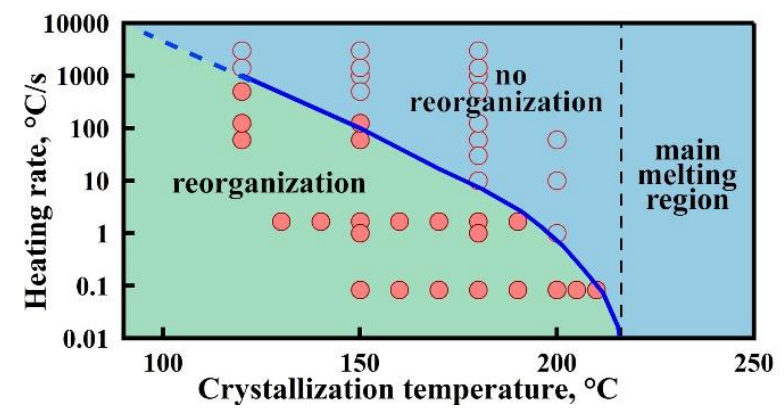

Figure 5. HR-CT-R diagram for isothermally melt-crystallized PTT. The border between the zones where the reorganization is present and absent corresponds to the critical heating rate.

In conclusion, based on a new technology of simultaneous millisecond time-resolved micro-focus X-ray scattering and ultra-fast chip scanning calorimetry we put forward a HeatingRate versus Crystallization-Temperature Reorganization Diagram. The main motivation of this work stems from the fact that the semicrystalline polymer structure can rapidly reorganize on heating. This drastically affects the measurable thermodynamic properties as they vary in the course of the run. The experimentalist would completely exclude such sample reorganization during the measurement but calorimetry alone cannot always discriminate the processes of reorganization from multiple melting. In contrast, the described on-time structural and thermal measurements using a MEMS-based platform make it possible to detect the processes of reorganization and identify their signature. The proposed HR-CT-R diagram can be established for any semicrystalline polymer in order to help designing thermal analysis experiments in which the one can rule out any uncontrolled evolution of the semicrystalline structure.

\section{ASSOCIATED CONTENT}

The Supporting Information is available free of charge on the ACS Publications website at DOI: .

Materials, instrumentation, data reduction and analysis (PDF).

\section{AUTHOR INFORMATION}

${ }^{\dagger}$ Authors equally contributed to this work

\section{Corresponding Author}

* Dimitri A. Ivanov: dimitri.ivanov@uha.fr

* Martin Rosenthal: martin.rosenthal@esrf.fr

\section{ORCID}

Alexey P. Melnikov: 0000-0003-2277-9644

Martin Rosenthal: 0000-0001-6014-6050

Dimitri A. Ivanov: 0000-0002-5905-2652

\section{Notes}

The authors declare no competing financial interest

\section{ACKNOWLEDGMENT}

The author greatly thanks the Ministry of education and science of the Russian Federation (contract \# 14.616.21.0072 from 28 July 2016 (RFMEFI61616X0072)) for financial support. The authors acknowledge excellent technical support provided by D. Hermida at the BM26B beamline of ESRF (Grenoble).

\section{ABBREVIATIONS}


SAXS, Small-angle X-ray scattering; WAXS, Wide-angle X-ray scattering, DSC, Differential scanning calorimetry.

\section{REFERENCES}

(1) Strobl, G. The Physics of Polymers: Concepts for Understanding Their Structures and Behavior. Springer-Verlag Berlin Heidelberg, 2007. DOI: doi.org/10.1007/978-3-540-68411-4.

(2) D. A. Ivanov, "1.11 Crystalline polymers" in: Comprehensive Polymer Science Encyclopedia, Edt. By F. Kremer, A. Khokhlov and M. Moeller. Elsevier. 2012 ISBN: 9780444533494.

(3) Holdsworth, P.J.; Turner-Jones, A. The melting behaviour of heat crystallized poly(ethylene terephthalate). Polymer 1971, 12, 195208.

(4) Groeninckx, G.; Reynaers, H. Morphology and melting behavior of semicrystalline poly(ethylene terephthalate). II. Annealed PET. Journal of Polymer Science Part B: Polymer Physics 1980, 18(6), 1325-1341.

(5) Yagfarov, M.Sh.; Mitrofanova, E.P. Recrystallization and secondary crystallization in polymers. Polymer Science USSR 1986, 28(5), 1011-1017.

(6) Bassett, D.C.; Olley, R.H.; Al Raheil, I.A.M. On Crystallization Phenomena in PEEK. Polymer 1988, 29, 1745-1754.

(7) Lee, Y.; Porter, R.S. Double-melting behavior of poly(ether ether ketone). Macromolecules 1987, 20, 1336-1341.

(8) Ivanov, D.A.; Jonas, A.M. Isothermal growth and reorganization upon heating of a single poly(aryl-ether-ether-ketone) (PEEK) spherulite, as imaged by atomic force microscopy". Macromolecules 1998, $31,4546-4550$.

(9) Ivanov, D.A.; Jonas, A.M.; Legras, R. The crystallization of poly(aryl-ether-ether-ketone) (PEEK). Reorganization processes during gradual reheating of cold-crystallized samples. Polymer 2000, 41 3719-3727.

(10) Huang, J.-M.; Ju, M.-Y.; Chu, P.P.; Chang, F.-C. Crystallization and melting behaviors of poly(trimethylene terephthalate). Journ. Polym. Res. 1999, 6(4), 259-266.

(11) Srimoaon, P.; Dangseeyun, N.; Supaphol, P. Multiple melting behavior in isothermally crystallized poly(trimethylene terephthalate). Eur. Polym. J. 2004, 40, 599-608.

(12) Chan, L.C.; Nae, H.N.; Gillham, J.K. Time-TemperatureTransformation (TTT) Diagrams of High Tg Epoxy Systems: Competition Between Cure and Thermal Degradation. Journ. Appl. Polym. Sci. 1984, 29, 33074327.

(13) Wisanrakkit, G.; Gillham, J.K. Continuous Heating Transformation (CHT) Cure Diagram of an Aromatic Amine/ Epoxy System at Constant Heating Rates. Journal of Applied Polymer Science 1991, 42 , 2453-2463.

(14) Nürnberger, F.; Grydin, O.; Schaper, M.; Bach, F.-W.; Koczurkiewicz, B.; Milenin, A. Microstructure Transformations in Tempering Steels during Continuous Cooling from Hot Forging Temperatures. Steel Research Int. 2010, 81(3), 224-233.

(15) Lu, K.; Lück, R.; Predel, B. Temperature vs. heating rate transformation diagram for a transition from the amorphous to the nanocrystalline state. Journal of Alloys and Compounds 1993, 201, 229-234.

(16) Lai, S.L.; Ramanath, G.; Allen, L.H. High-speed $\left(10^{4}{ }^{\circ} \mathrm{C} / \mathrm{s}\right)$ scanning microcalorimetry with monolayer sensitivity $\left(\mathrm{J} / \mathrm{m}^{2}\right)$. Appl. Phys. Lett. 1995, 67, 1229-1231.
(17) "Design of an in-situ setup combining nanocalorimetry and nano- or micro-focus X-ray scattering to address fast structure formation processes" M. Rosenthal, A.P. Melnikov, A.A. Rychkov, D. Doblas, D.V. Anokhin, M. Burghammer, and D.A. Ivanov. In: Fast Scanning Calorimetry edt. by C. Schick and V. Mathot, Springer International Publishing, Switzerland 2016, ISBN 978-3-319-31327-6.

(18) Rosenthal, M.; Doblas, D.; Hernandez, J.J.; Odarchenko, Ya.I. Burghammer, M.; Di Cola, E.; Spitzer, D.; Antipov, A.E.; Aldoshin, L.S.; D.A. Ivanov. High-Resolution Thermal Imaging with a Combination of Nano-Focus X-ray Diffraction and Ultra-Fast Chip Calorimetry. Journal of Synchrotron Radiation 2014, 21, 223-228.

(19) Melnikov, A.P.; Rosenthal, M.; Rodygin, A.I.; Doblas, D.; Anokhin, D.V.; Burghammer, M.; Ivanov, D.A. Re-exploring the double-melting behavior of semirigid-chain polymers with an in-situ combination of synchrotron nano-focus X-ray scattering and nanocalorimetry. Eur. Polym. J. 2016, 81, 598-606.

(20) Riekel, C.; Di Cola, E.; Burghammer, M.; Reynolds, M.; Rosenthal, M.; Doblas, D.; Ivanov, D.A. Thermal Transformations of SelfAssembled Gold Glyconanoparticles Probed by Combined Nanocalorimetry and X-ray Nanobeam Scattering. Langmuir 2015, 31, 529-534.

(21) Rosenthal, M.; Melnikov, A.P.; Burghammer, M.; Ivanov, D.A. Reorganization of semicrystalline polymers on heating: analyzing common misconceptions in the interpretation of calorimetric data. Response on the "Comment on "Re-exploring the double-melting behavior of semirigid-chain polymers with an in-situ combination of synchrotron nanofocus X-ray scattering and nanocalorimetry" by Dimitri A. Ivanov et al. [European Polymer Journal 81 (2016) 598-606.]". Eur. Polym. J. 2017, 94, 517-523.

(22) G. Vigier, J. Tatibouet, A. Benatmane, R. Vassoille, Amorphous phase evolution during crystallization of poly(ethylene-terephthalate), Colloid and Polym. Sci. 1992, 270(12), 1182-1187.

(23) Ivanov, D.A.; Legras, R.; Jonas, A.M. The crystallization of poly(aryl-ether-ether-ketone) (PEEK). Interdependence between the evolution of amorphous and crystalline regions during isothermal coldcrystallization, Macromolecules 1999, 32, 1582-1592.

(24) Ivanov D.A., Hocquet S., Dosiére M., Koch M.H.J. Exploring the melting of a semirigid-chain polymer with temperature-resolved small-angle X-ray scattering. Eur. Phys. J. E. 2004, 13, 363-378.

(25) Ivanov, D.A.; Bar, G.; Dosière, M.; Koch, M.H.J. A Novel View on Crystallization and Melting of Semirigid Chain Polymers: The Case of Poly(trimethylene terephthalate). Macromolecules 2008, 41, 9224-9231.

(26) Rosenthal, M.; Portale, G.; Burghammer, M.; Bar, G.; Samulski, E.T.; Ivanov, D.A. Exploring the origin of crystalline lamella twist in semi-rigid chain polymers: the model of Keith and Padden revisited. Macromolecules 2012, 45(18), 7454-7460.

(27) Rosenthal, M.; Burghammer, M.; Bar, G.; Samulski, E.T.; Ivanov, D.A. Switching Chirality of Hybrid Left-Right Crystalline Helicoids Built of Achiral Polymer Chains: When Right to Left Becomes Left to Right. Macromolecules 2014, 47(23), 8295-8304.

(28) Rosenthal M.; Ivanov D.A. Microstructure of Banded Polymer Spherulites: New Insights from Synchrotron Nanofocus X-Ray Scattering. Adv. Polym. Sci. 2017, 277, 95-126. 


\section{What thermal analysis can tell us about melting of semicrystalline}

polymers: exploring the general validity of the techniqueThermal Analysis of Semicrystalline Polymers: Exploring the General Validity of the Technique

Alexey P. Melnikov, ${ }^{\dagger, a, b}$ Martin Rosenthal, ${ }^{\dagger, *, c}$ and Dimitri A. Ivanov",a,b,d

${ }^{a}$ Lomonosov Moscow State University, Faculty of Fundamental Physical and Chemical Engineering, GSP-1, 1-51 Leninskie Gory, Moscow, Russian Federation;

${ }^{\mathrm{b}}$ Moscow Institute of Physics and Technology (State University), Institutskiy per. 9, Dolgoprudny, Russian Federation;

${ }^{\mathrm{c}}$ European Synchrotron Radiation Facility (ESRF), 6 rue Jules Horowitz, 38043 Grenoble, France

${ }^{\mathrm{d}}$ Institut de Sciences de Matériaux de Mulhouse-IS2M, CNRS UMR 7361, Jean Starcky, 15, F-68057 Mulhouse, France.

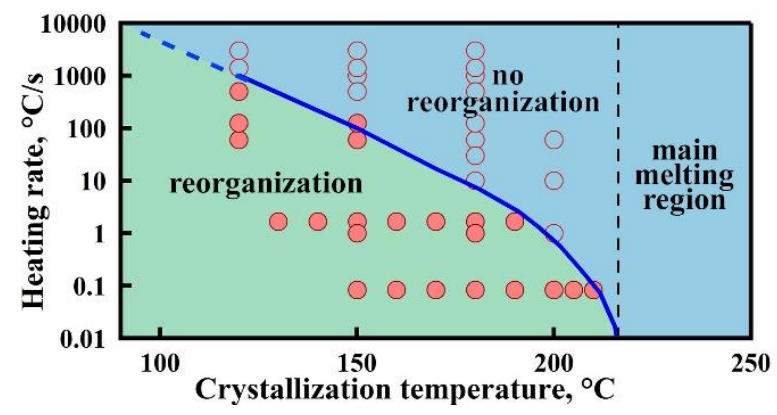

